\title{
On-tissue Derivatization Via Electrospray Deposition for Matrix-Assisted Laser Desorption/Ionization Mass Spectrometry Imaging of Endogenous Fatty Acids in Rat Brain Tissues
}

\author{
Qian Wu, Troy J. Comi, Bin Li, Stanislav S. Rubakhin, and Jonathan V. Sweedler \\ Department of Chemistry and the Beckman Institute, University of Illinois at \\ Urbana-Champaign, Urbana, Illinois 61801, United States
}

\section{Supporting Information}

Table of Contents

Additional Experimental Section S-2

Figure S1. Schematic illustration of sample preparation process and devices S-3

Figure S2. MALDI mass spectra of rat cerebrum tissue S-4

Figure S3. Effects of various electrospray parameters on MALDI MS detection S-5

Figure S4. Dependence of MALDI MSI output on electrospray flowrate S-6

Figure S5. MALDI MS/MS spectra of FFAs derivatives

Figure S6. Mass chromatograms of DHA standard derivative and rat brain tissue punchesS-8

Figure S7. Reproducibility of the method S-10

Figure S8. Comparison of MALDI MSI results ..S-11

Figure S9. MS/MS spectra of different lipids S-12

Figure S10. PCA score and loading plots of lipid peaks in rat cerebrum tissue S-13

Figure S11. Comparison of MALDI MSI and LC-MS/MS ..... S-14

Figure S12. Relative recovery of spiked standard in rat brain tissue after derivatization $\quad . . S-15$

Table S1. MRM parameters of LC-MS/MS for fatty acids standard S-16 


\section{Additional Experimental Section}

Details of Sample Preparation from the Four Animals. From animal 1, a total of 10 coronal tissue sections containing hippocampal regions were prepared and used for derivatization optimization and comparison between airbrush and electrospray deposition. Tissues from animal 2 were imaged using the optimized conditions; three coronal sections were collected from the same sagittal direction as animal 1 and used for MALDI MSI at a $20-\mu \mathrm{m}$ pixel width. In addition, several punches from this animal were harvested to collect specific brain sub-regions for LC-MS/MS validation, as described in the manuscript. Animals 3 and 4 were used to test the reproducibility of the optimized approach, each sampled on a different month, by taking three coronal brain slices from the same region as animal 2.

Laboratory-constructed Sublimation Chamber. Briefly, an aluminum foil boat was affixed with double-sided conductive copper tape to the inner base of the sublimation chamber. Samples were attached to a copper plate affixed to the bottom face of an ice-filled cold finger. The MALDI matrix-to-sample distance was $\sim 20 \mathrm{~mm}$. For each sublimation deposition, $350 \mathrm{mg}$ of powdered 2,5-dihydroxybenzoic acid was distributed evenly in the boat. The sublimation chamber and cold finger were assembled together per manufacturer's instructions, pumped to intermediate vacuum ( $\sim 10$ mTorr), and placed in a heating mantle (Glas-Col LLC, Terre Haute, IN) to equilibrate the vacuum and cool the sample plate. The optimized deposition conditions for derivatized samples included supplying $120 \mathrm{~V}$ to the heating mantle for $12 \mathrm{~min}$. After matrix deposition, the chamber was removed from the mantle, vented with room temperature air $(25$ ${ }^{0} \mathrm{C}$ ), and the sample was promptly removed from the cold finger.

LC Separation Conditions. Analytes were separated on an Acclaim ${ }^{\mathrm{TM}}$ mixed mode HILIC-1 LC column $(3 \mu \mathrm{m}, 150 \mathrm{~mm} \times 2.1 \mathrm{~mm}$ inner diameter; Thermo Scientific, Waltham, MA). Separation started with an isocratic step, $10 \%$ mobile phase B $(50 \% \mathrm{ACN}, 40 \%$ water, and $10 \%$ $50 \mathrm{mM}$ ammonium-acetate buffer, $\mathrm{pH} 4)$ and $90 \%$ phase $\mathrm{A}(90 \% \mathrm{ACN}$ and $10 \% 50 \mathrm{mM}$ ammonium-acetate buffer, $\mathrm{pH} 4$ ), followed by a 5 min gradient from $10 \%$ phase $\mathrm{B}$ to $100 \%$ phase $\mathrm{B}$ at a flow rate of $0.375 \mathrm{~mL} / \mathrm{min}$ at room temperature $\left(25{ }^{0} \mathrm{C}\right)$. The 2-picolylamine derivatives of the fatty acids were analyzed by electrospray ionization-MS in the positive-ion mode.

MS Conditions for LC-MS/MS Detection. The MS conditions were as follows: 65 psi nebulizer gas pressure, $15 \mathrm{~L} / \mathrm{min}$ cone gas flow, $+4500 \mathrm{~V}$ spray voltage, $150{ }^{0} \mathrm{C}$ cone temperature, and $450{ }^{\circ} \mathrm{C}$ heated probe temperature. Argon was used as a collision gas in the multiple reaction monitoring (MRM) mode with a pressure of $1.5 \mathrm{mTorr}$. The MRM parameters, including ion transitions (precursor and product ions) and collision energies for each analyte, are described in Table S1.

Quantitation of LC-MS/MS Data. Fatty acids were identified by both retention time and specific MRM transitions. External standard calibration was used to quantitate the fatty acids. The relative recoveries of external standards used to calibrate different fatty acids were near $100 \%$ (Figure S11). A two-sample $t$-test was also applied to triplicates of the LC-MS data, and 
the $p$-values of every pair were false discovery rate-corrected with a Benjamini and Hochberg's FDR-controlling procedure with $c(N)=1$.

(A)

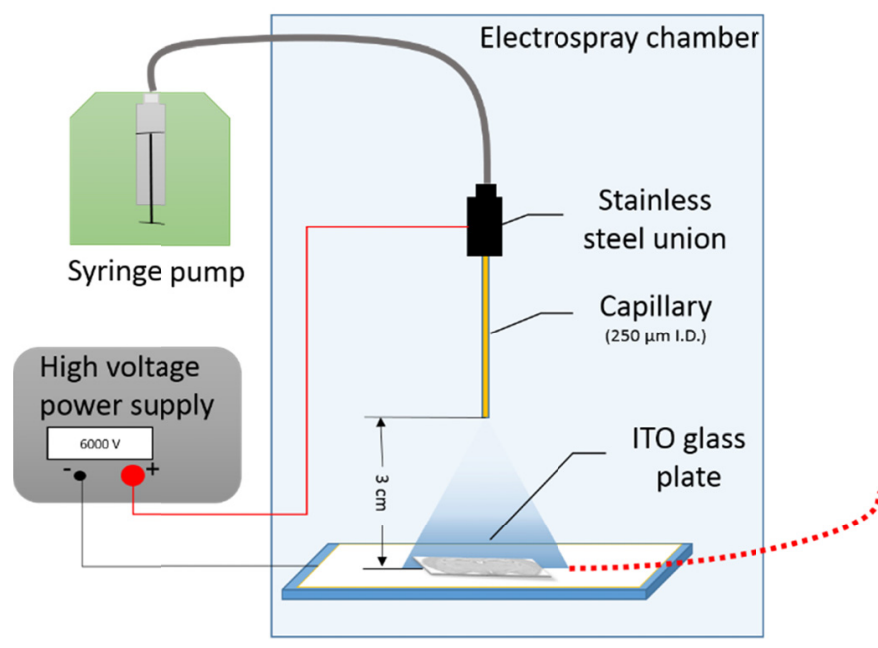

On-tissue derivatization with electrospray
(B)

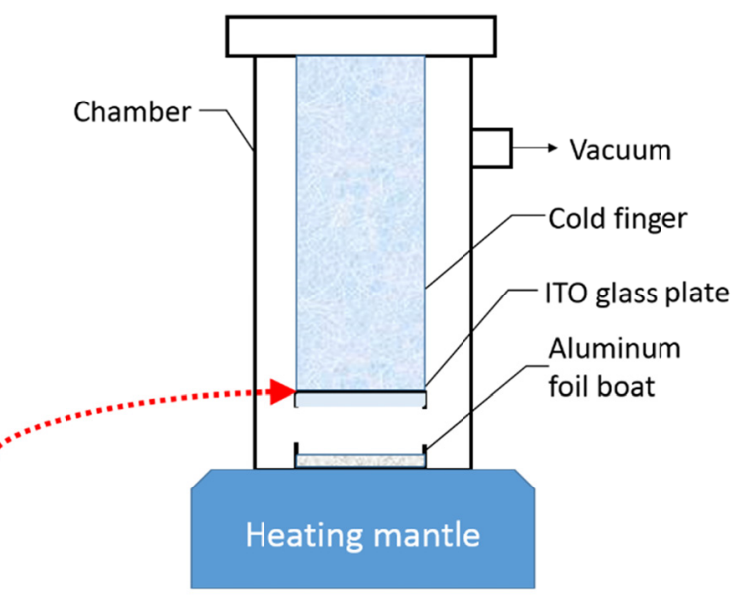

Matrix deposition with sublimation

Figure S1. Schematic illustration of the sample preparation process and devices. (A) Derivatization reagent is applied in a laboratory-constructed electrospray chamber. (B) After derivatization, the sample is transferred to a sublimation chamber for MALDI matrix application. 
(A)

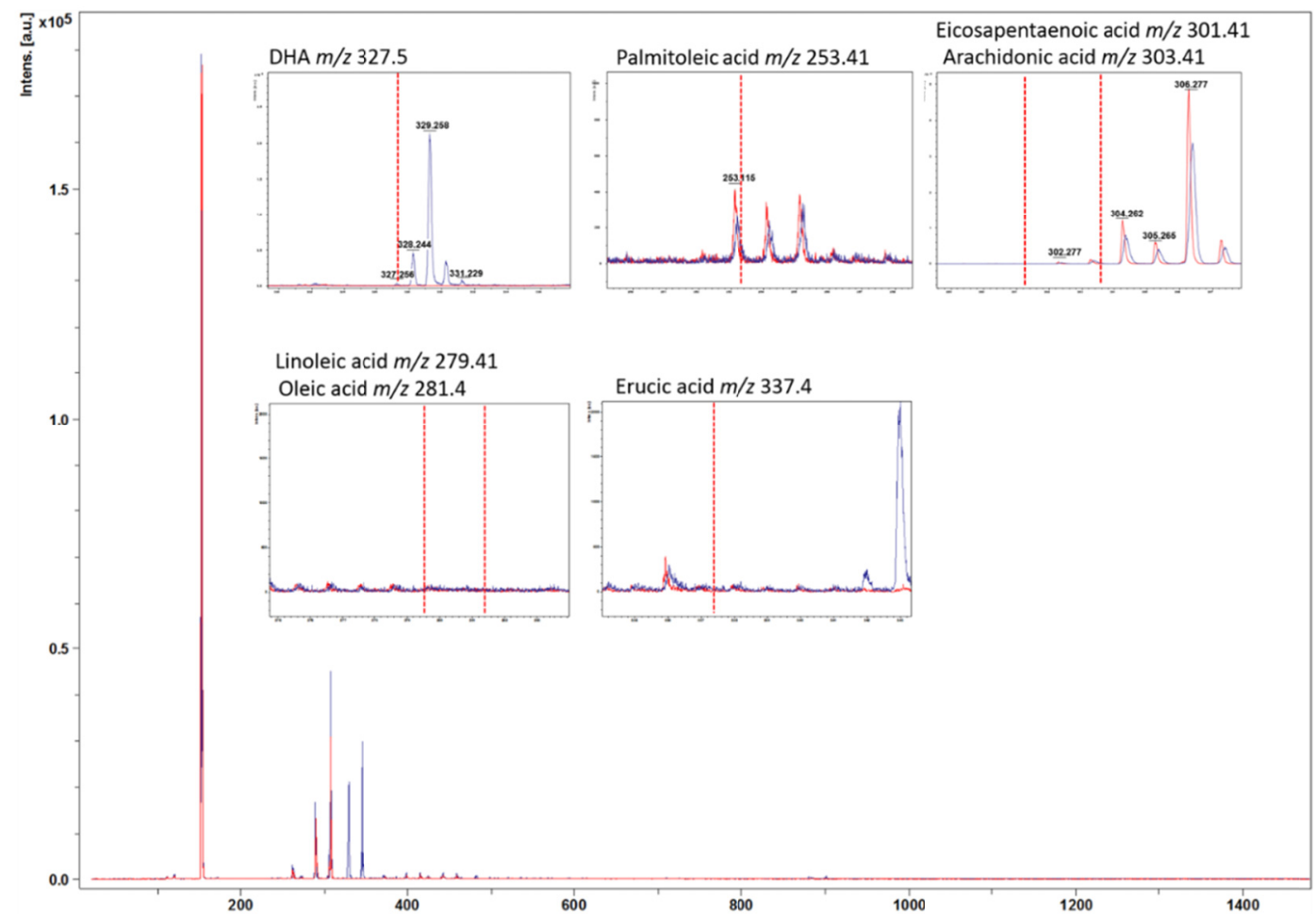

(B)

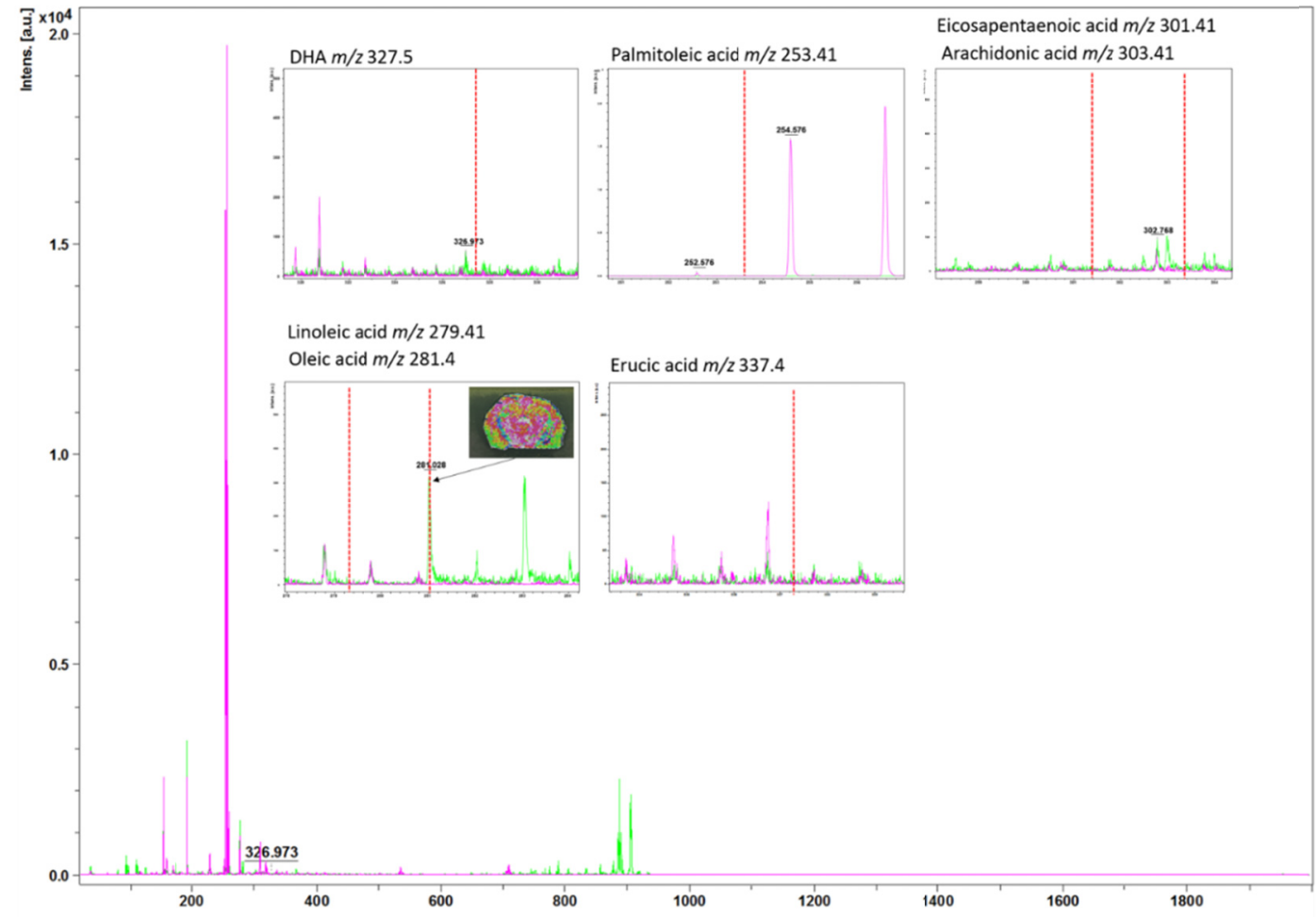

Figure S2. MALDI mass spectra of rat cerebrum tissue (blue and green) and blank plate (red and pink) without derivatization using the matrices (A) DHB and (B) 9-AA in negative mode. 

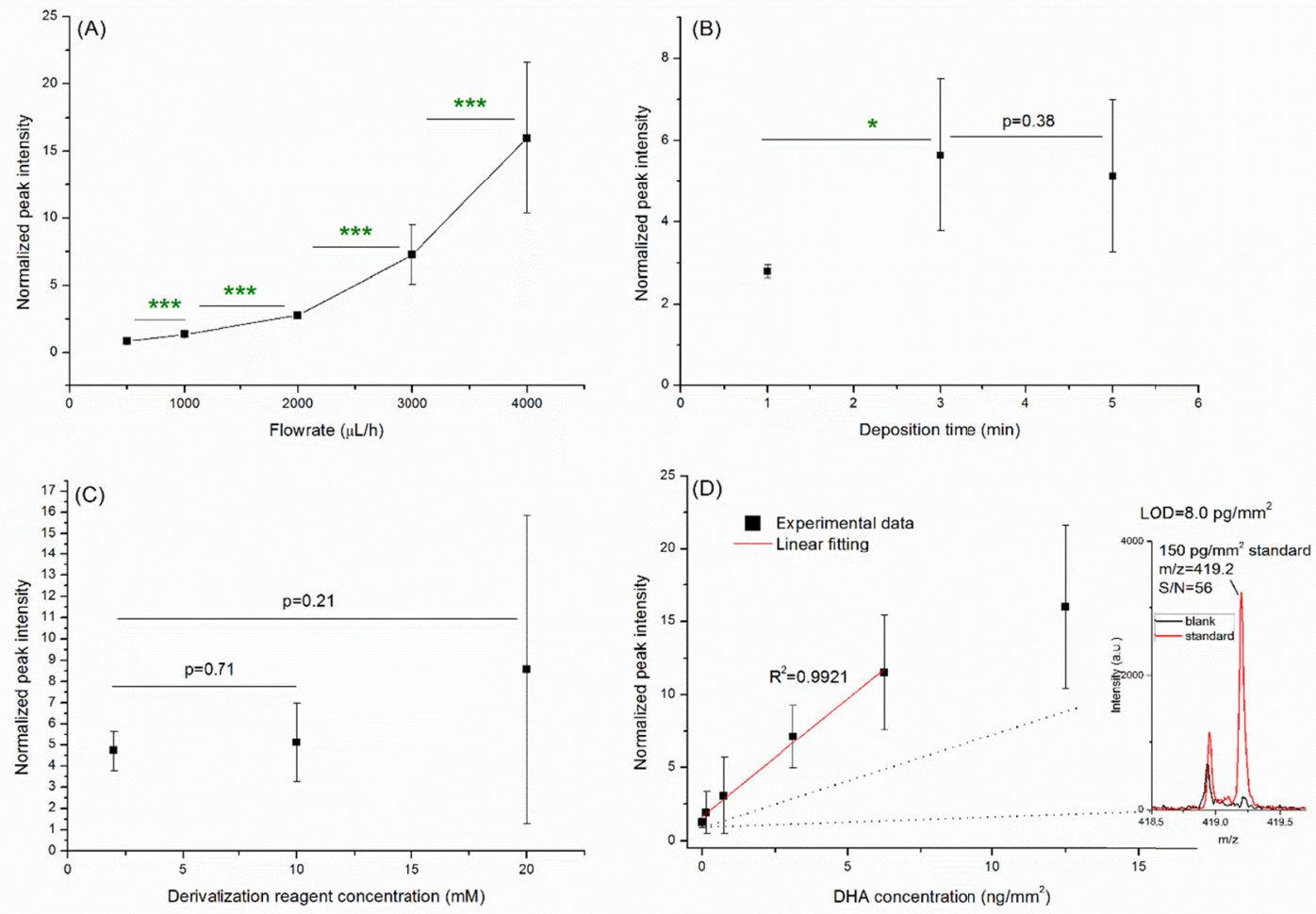

Figure S3. Effects of various electrospray derivatization agent deposition parameters on MALDI MS detection of DHA standard. The following electrospray conditions were optimized: (A) reagent solution flowrate, (B) reagent deposition time, and (C) derivatization reagent concentration. (D) DHA calibration curve obtained with the optimized deposition of reagent solution via electrospray. $\mathrm{P}$ values were calculated by the two sample $t$-test. Error bars represent standard deviation. The deposited concentration of DHA standard in solution used in A, B, and C was $17.5 \mathrm{ng} / \mathrm{mm}^{2}$. PA concentration in (D) was $2 \mathrm{mM}$. Deposition time for (A) was set to keep the total deposited volume equal to $66 \mu \mathrm{L}$, and for (C) and (D) was $3 \mathrm{~min}$. Limit of detection (LOD) was calculated by average peak height of $0.15 \mathrm{ng} / \mathrm{mm}^{2}$ with $\mathrm{S} / \mathrm{N}=3$. $*$ and $* * *$ indicate $p$ values of $<0.05$ and $<0.001$, respectively. Mean and standard deviation are presented. 


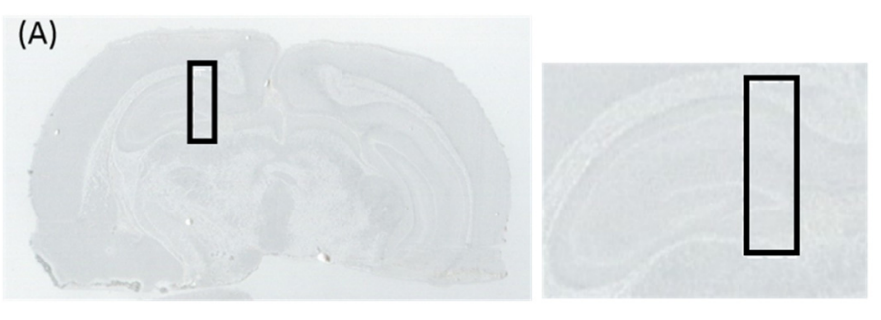

(B-1)

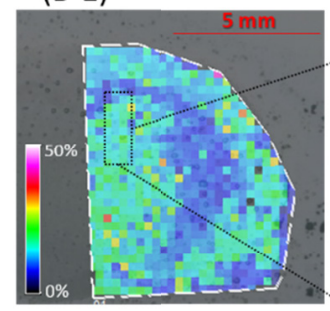

(B-2)

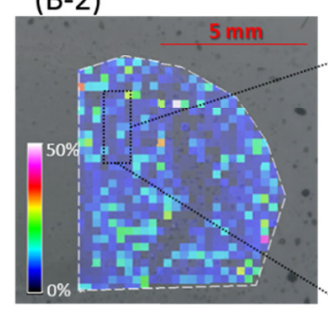

(C-1)

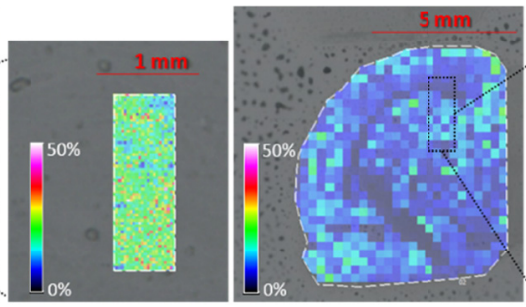

(C-2)
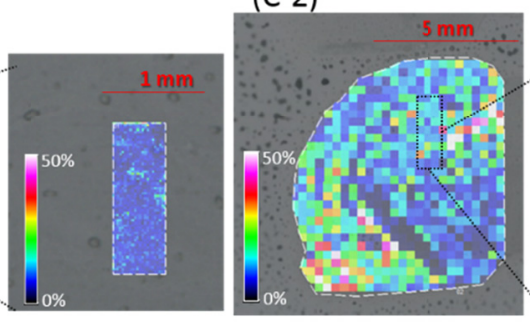

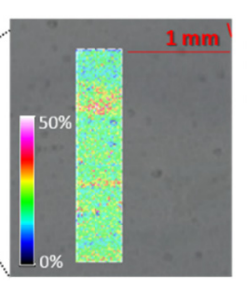

(D-1)
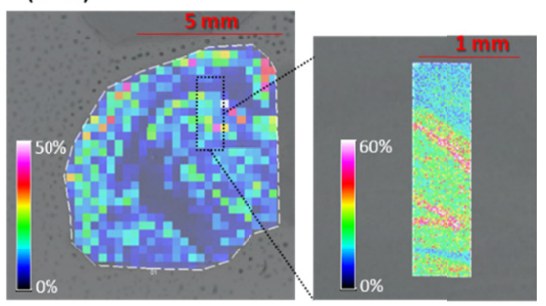

$(\mathrm{D}-2)$
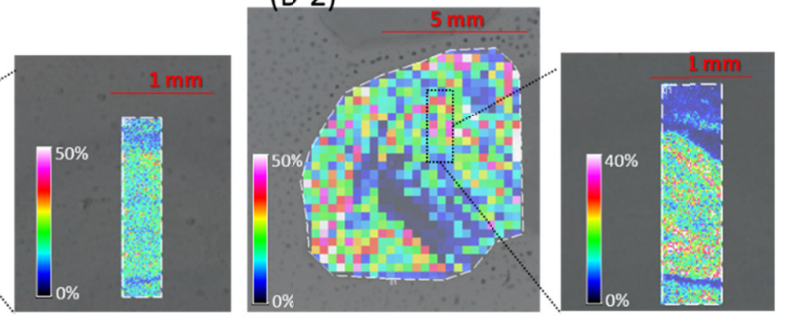

Figure S4. Dependence of MALDI MSI output on the flowrate of the electrospray-deposited derivatization solution. (A) Optical images of (left) $10 \mu \mathrm{m}$ coronal rat brain slice and (right) its hippocampal region. (Boxed areas in Panels A indicate the hippocampal area and correspond to the boxes in Panels B-E.) (B-D) MALDI MSI images of derivatized: (1) DHA-like and (2) AA-like molecular distributions in samples prepared using electrospray deposition. Different flow rates for the derivatization agent were tested: (B) $4 \mathrm{~mL} / \mathrm{h}$; (C) $3 \mathrm{~mL} / \mathrm{h}$; (D) $2 \mathrm{~mL} / \mathrm{h}$. 

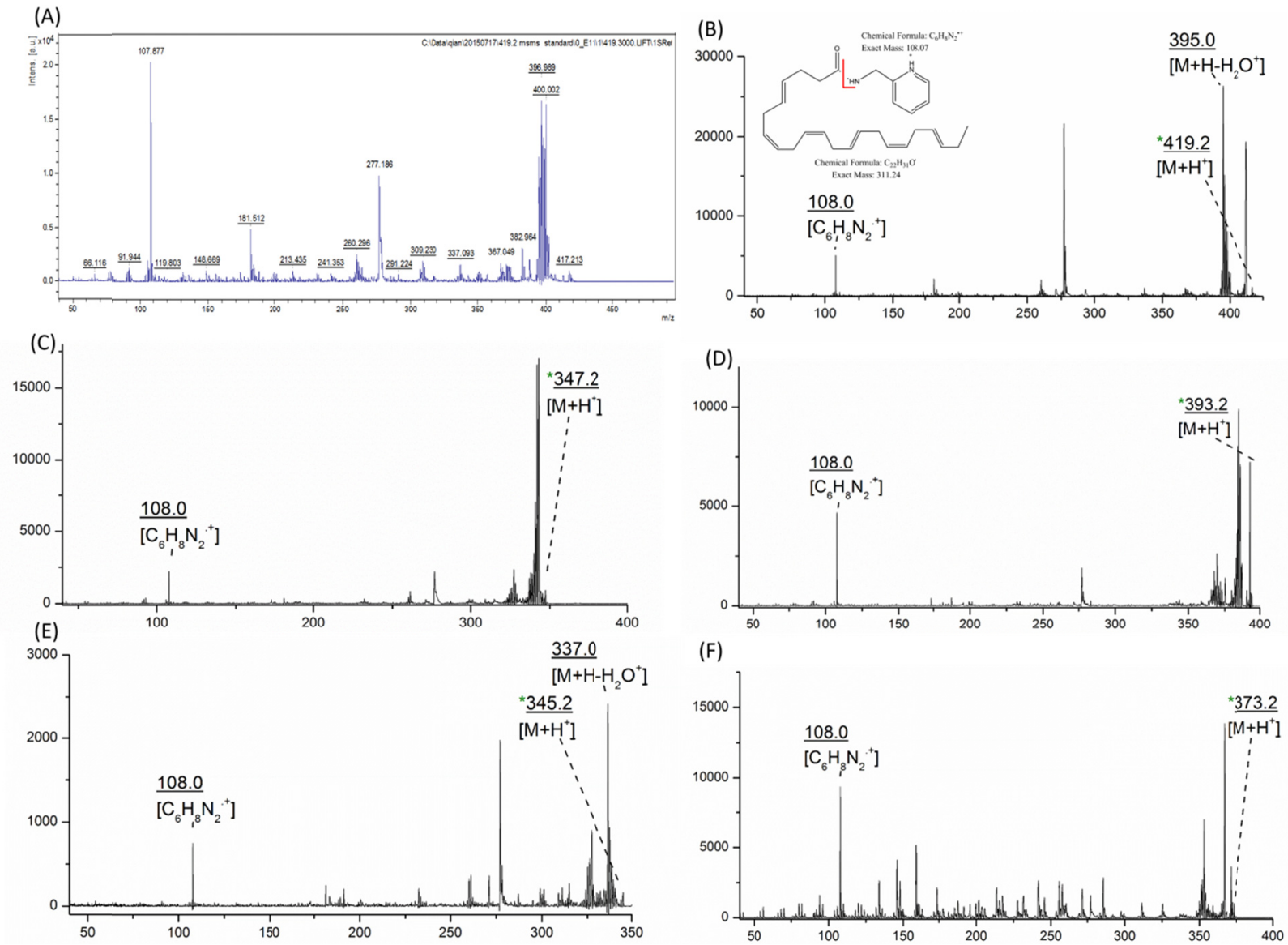

Figure S5. MALDI MS/MS spectra of (A) DHA standard and from the same tissue used to confirm the identities of the FFAs (B-F). B, DHA; C, palmitic acid; D, eicosapentaenoic acid; E, palmitoleic acid; F, oleic acid. 


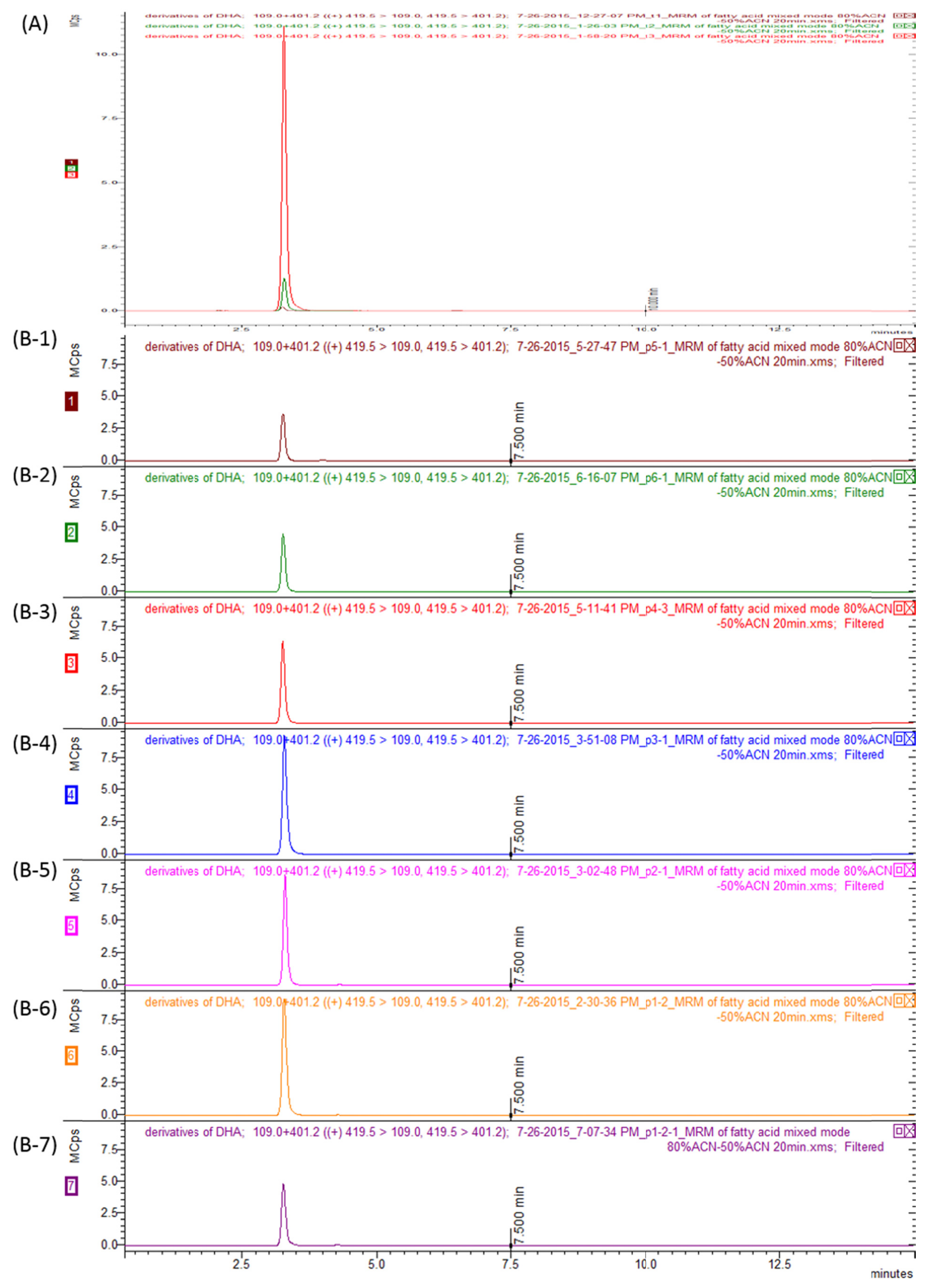


Figure S6. Mass chromatograms of DHA standard derivative with different concentrations (A, 10ng/mL, 100ng/mL and $1000 \mathrm{ng} / \mathrm{mL}$ ) and rat brain tissue punches (B-1, region 5, B-2, region 6, B-3, region 4, B-4, region 3, B-5, region 2, B-6, region 1, B-7, region 7) 
Rat 1

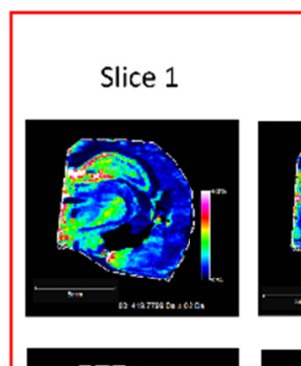

(B)

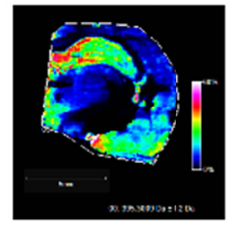

(c)

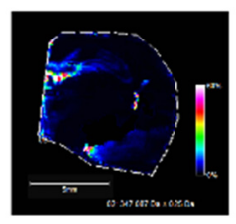

(D)

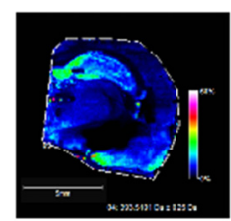

(E)
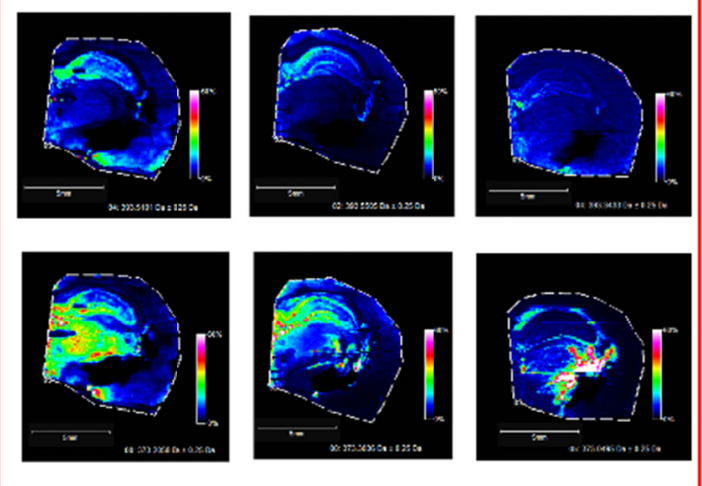

Rat 2
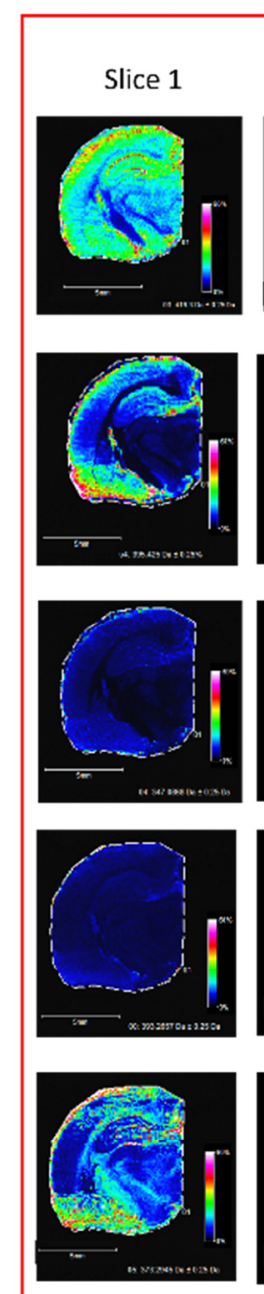
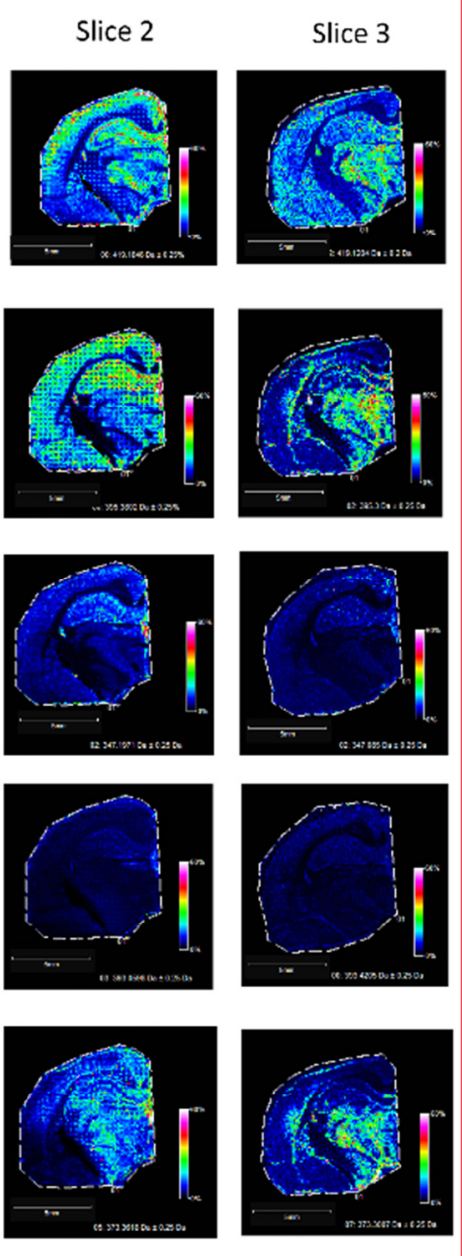
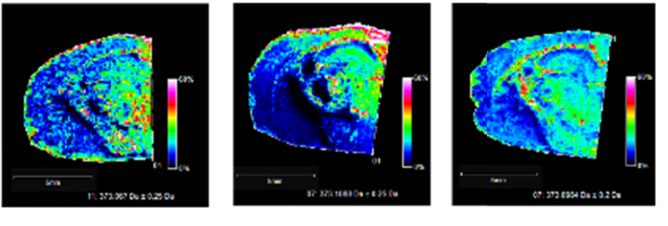

Figure S6. Heat maps of (A) DHA, (B) AA, (C) palmitic acid, (D) eicosapentaenoic acid, and (E) oleic acid in different rat cerebrum tissues (rats 1, 2 and 3 correspond to animals 3, 2 and 4 in the Experimental section), measured in different months with on-tissue derivatization MALDI MSI. Intensity bars are $0 \%-60 \%$, and scale bars are $5 \mathrm{~mm}$; the signal of eicosapentaenoic acid for rat 3 is too low to see its distribution. 
(A)

DHA
$m / z 419.3$

AA

$m / z 395.3$
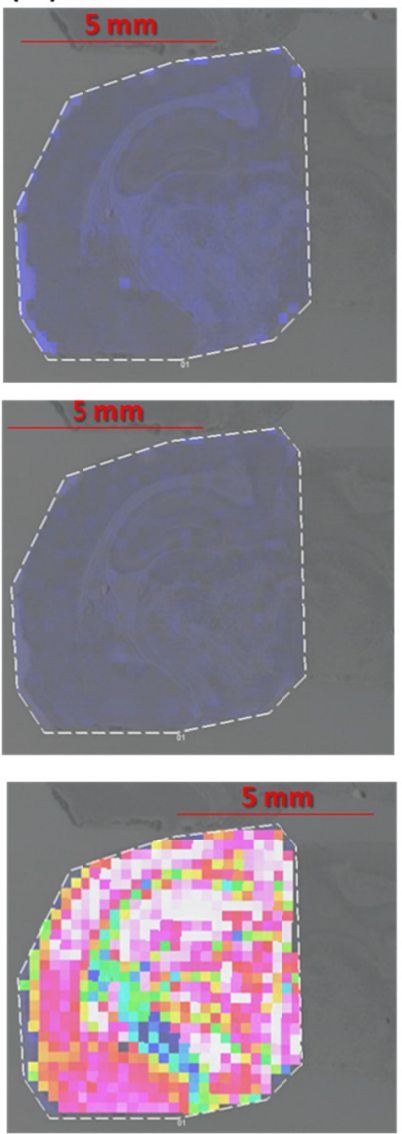

PC (40:6)

$m / z 872.6$
(B)
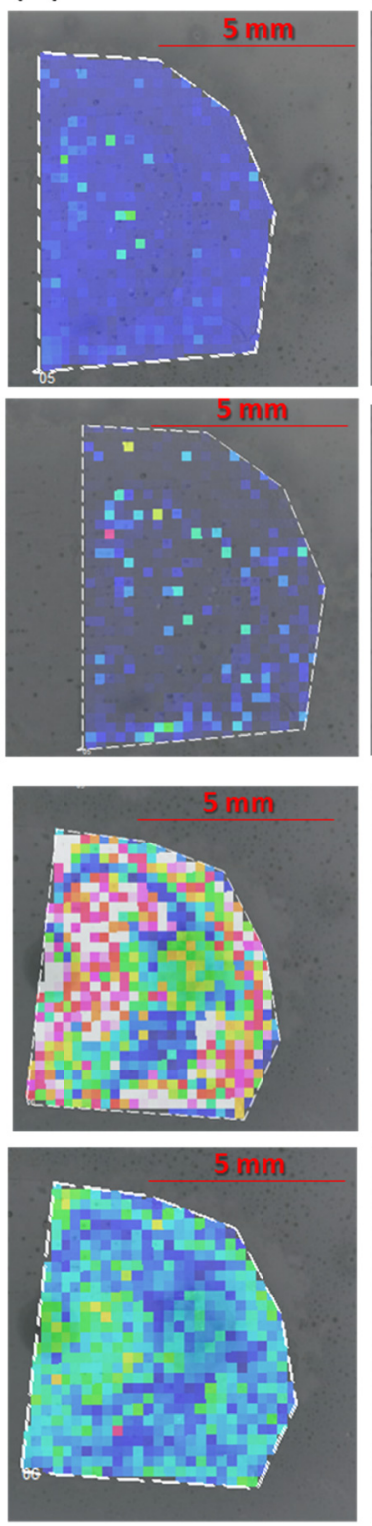

(C)
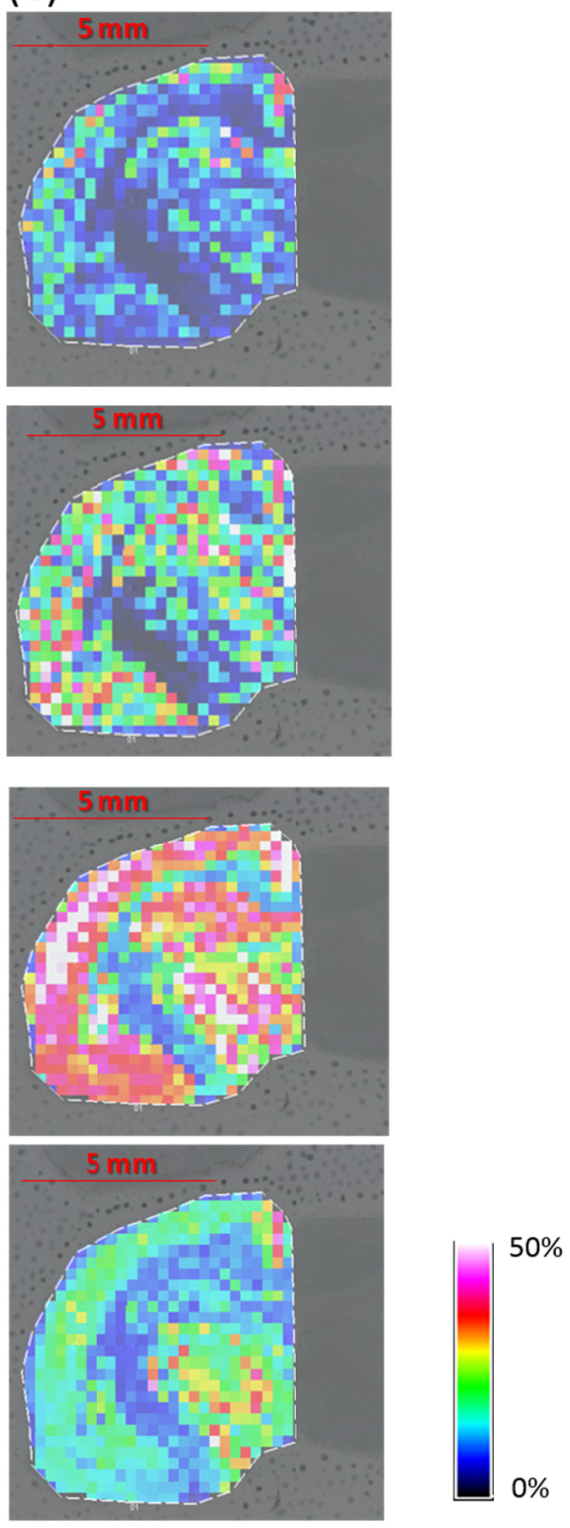

Figure S7. Comparison of MALDI MSI results of fatty acids and lipids (A) without derivatization, and with derivatization via (B) airbrush and (C) electrospray deposition. 

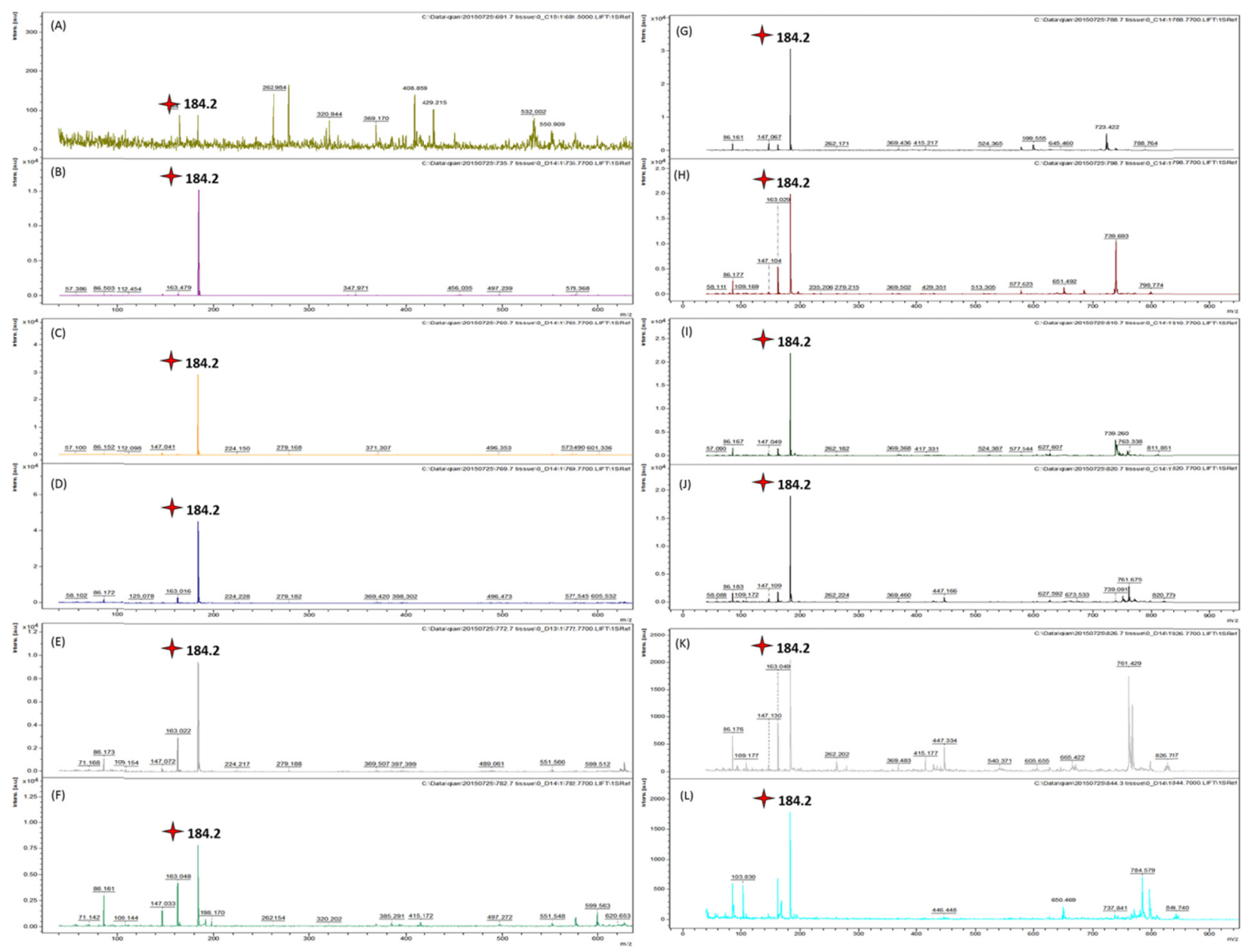

Figure S8. MALDI MS/MS spectra of different lipids: (A) $\mathrm{m} / z$ 691.5; (B) $\mathrm{m} / \mathrm{z} 735.7$; (C) $\mathrm{m} / \mathrm{z} 760.7$; (D) $m / z$ 769.7; (E) $m / z$ 772.7, (F) $m / z$ 782.7; (G) $m / z$ 788.7; (H) $m / z$ 798.7; (I) $m / z ~ 810.7$; (J) $m / z ~ 820.7$; (K) $m / z$ 826.7; (L) $m / z$ 844.6. 

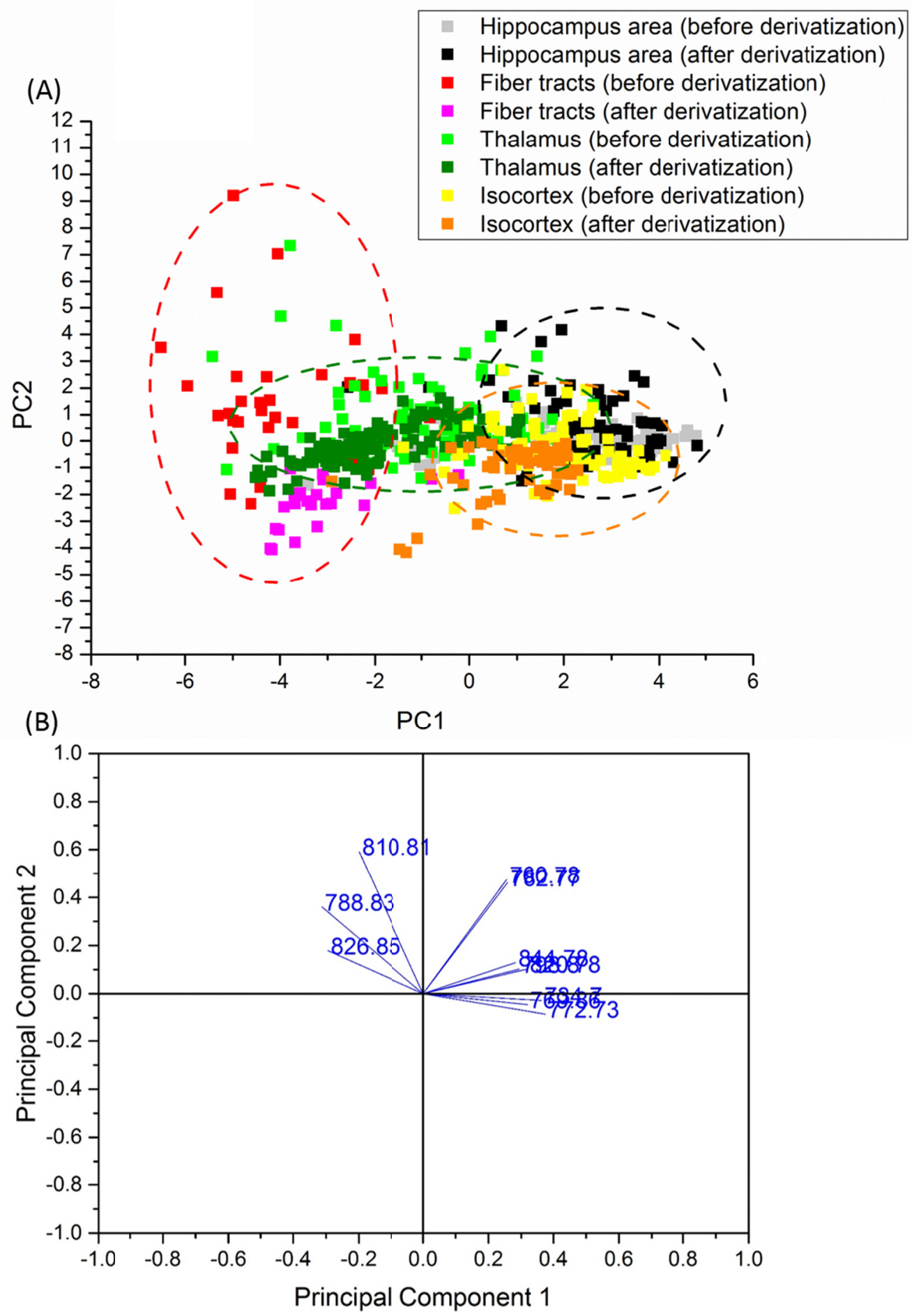

Figure S9. PCA (A) score plot and (B) loading plot of lipid peaks in different regions of rat cerebrum tissue. The dashed circles were drawn to show the separated distribution of different cerebrum regions; red circle, fiber tracts; green circle, thalamus; black circle, hippocampus; orange circle, isocortex. 


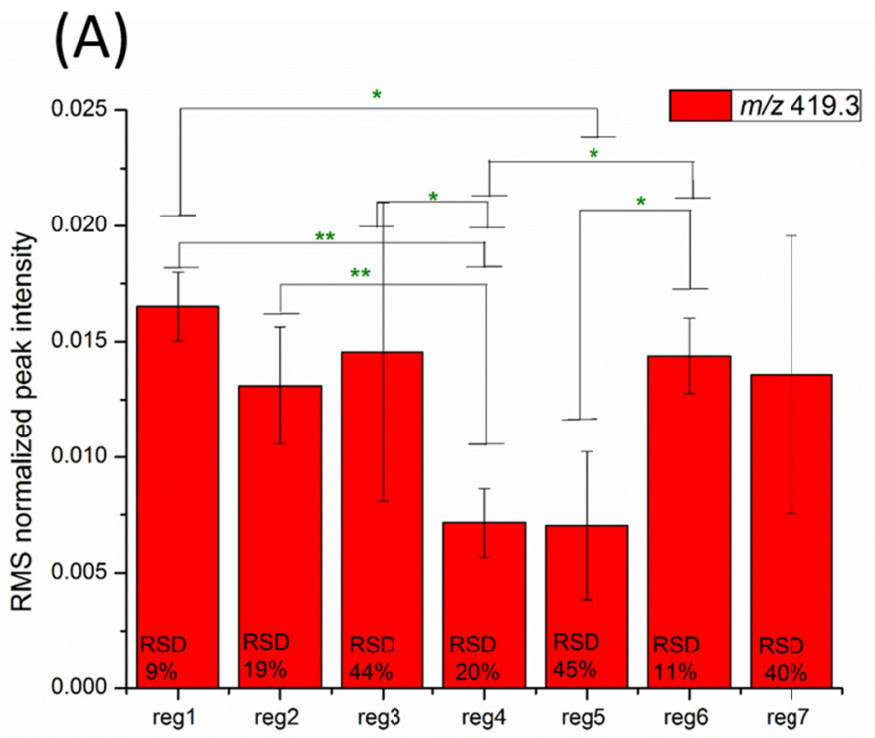

(B)

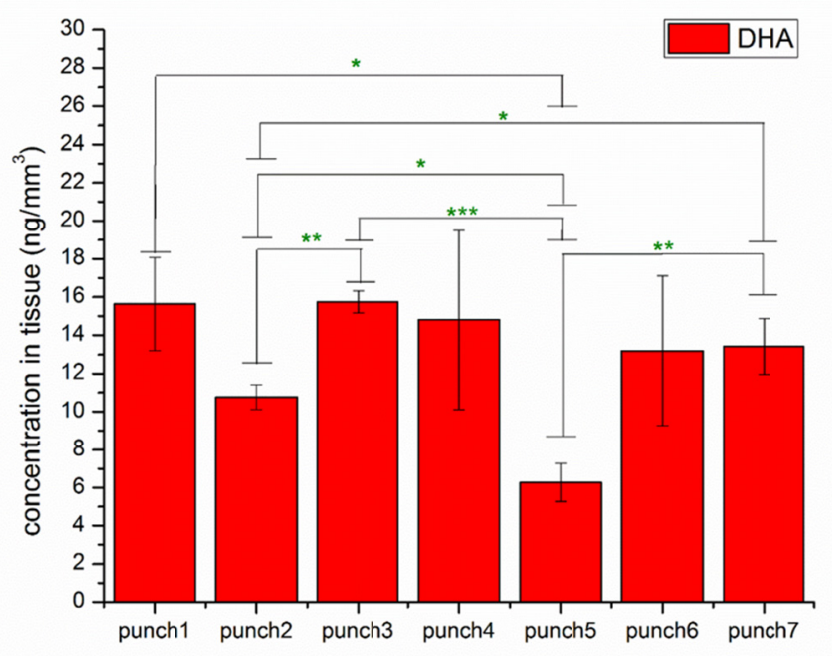

Figure S10. (A) Average peak intensity of MALDI MSI $(n=3)$ and (B) quantitation results of LCMS/MS from different cerebrum regions $(\mathrm{n}=3)$. P values were calculated by the two-sample $t$-test. *,**, and $* * *$ indicate $p$ values of $<0.05,<0.01$, and $<0.001$, respectively 


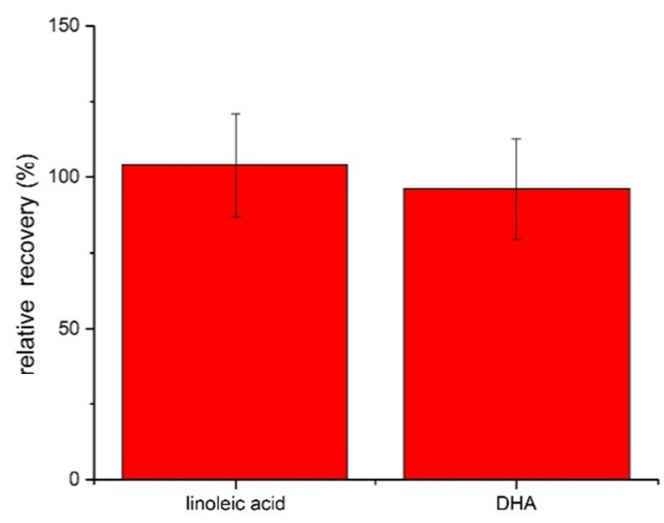

Figure S11. Relative recovery of spiked standard in rat brain tissue after derivatization with external standard calibration method. 
Table S1. MRM parameters for LC-MS/MS for fatty acids standard.

\begin{tabular}{|c|c|c|c|c|}
\hline Compounds & Precursor ion & Product ion & $\begin{array}{c}\text { Collision energy } \\
\text { (ev) }\end{array}$ & Scan time $(\%)$ \\
\hline \multirow{2}{*}{ DHA-PA } & \multirow{2}{*}{419.5} & 109.1 & 20 & 90 \\
\hline & & 401.1 & 20 & 10 \\
\hline \multirow{2}{*}{ Linolenic acid-PA } & \multirow{2}{*}{369.4} & 109.1 & 20 & 90 \\
\hline & & 350.8 & 20 & 10 \\
\hline
\end{tabular}

